Western University

Scholarship@Western

Brain and Mind Institute Researchers'

Publications

Brain and Mind Institute

6-1-2012

\title{
Greater benefits of multisensory integration during complex sensorimotor transformations.
}

\author{
Verena N Buchholz \\ Radboud University Nijmegen, Donders Institute for Brain, Cognition, and Behavior, Nijmegen, The \\ Netherlands \\ Samanthi C Goonetilleke \\ Departments of Physiology and Pharmacology Western University \\ W Pieter Medendorp \\ Radboud University Nijmegen, Donders Institute for Brain, Cognition, and Behavior, Nijmegen, The \\ Netherlands \\ Brian D Corneil \\ Drepartments of Physiology, Pharmacology and Psychology Western University \& The Brain and Mind \\ Institute Robarts Reseach Institute
}

Follow this and additional works at: https://ir.lib.uwo.ca/brainpub

Part of the Neurosciences Commons, and the Psychology Commons

\section{Citation of this paper:}

Buchholz, Verena N; Goonetilleke, Samanthi C; Medendorp, W Pieter; and Corneil, Brian D, "Greater benefits of multisensory integration during complex sensorimotor transformations." (2012). Brain and Mind Institute Researchers' Publications. 125.

https://ir.lib.uwo.ca/brainpub/125 


\title{
Greater benefits of multisensory integration during complex sensorimotor
}

\section{transformations}

\author{
Verena N. Buchholz, ${ }^{1}$ Samanthi C. Goonetilleke, ${ }^{2}$ W. Pieter Medendorp, ${ }^{1}$ and Brian D. Corneil ${ }^{2,3,4}$ \\ ${ }^{1}$ Radboud University Nijmegen, Donders Institute for Brain, Cognition, and Behavior, Nijmegen, The Netherlands; \\ Departments of ${ }^{2}$ Physiology and Pharmacology and ${ }^{3}$ Psychology, University of Western Ontario; and ${ }^{4}$ The Brain and Mind \\ Institute, Robarts Research Institute, London, Ontario, Canada
}

Submitted 23 December 2011; accepted in final form 22 March 2012

\begin{abstract}
Buchholz VN, Goonetilleke SC, Medendorp WP, Corneil BD. Greater benefits of multisensory integration during complex sensorimotor transformations. J Neurophysiol 107: 3135-3143, 2012. First published March 28, 2012; doi:10.1152/jn.01188.2011.-Multisensory integration enables rapid and accurate behavior. To orient in space, sensory information registered initially in different reference frames has to be integrated with the current postural information to produce an appropriate motor response. In some postures, multisensory integration requires convergence of sensory evidence across hemispheres, which would presumably lessen or hinder integration. Here, we examined orienting gaze shifts in humans to visual, tactile, or visuotactile stimuli when the hands were either in a default uncrossed posture or a crossed posture requiring convergence across hemispheres. Surprisingly, we observed the greatest benefits of multisensory integration in the crossed posture, as indexed by reaction time (RT) decreases. Moreover, such shortening of RTs to multisensory stimuli did not come at the cost of increased error propensity. To explain these results, we propose that two accepted principles of multisensory integration, the spatial principle and inverse effectiveness, dynamically interact to aid the rapid and accurate resolution of complex sensorimotor transformations. First, early mutual inhibition of initial visual and tactile responses registered in different hemispheres reduces error propensity. Second, inverse effectiveness in the integration of the weakened visual response with the remapped tactile representation expedites the generation of the correct motor response. Our results imply that the concept of inverse effectiveness, which is usually associated with external stimulus properties, might extend to internal spatial representations that are more complex given certain body postures.
\end{abstract}

multisensory integration; oculomotor; reference frames

THE ABILITY TO PROCESS SENSORY information such as sound, touch, and vision in an integrated fashion is crucial to survival. This process, called multisensory integration, allows for a coherent representation of the environment, enabling faster and more contextually appropriate responses (Stein and Meredith 1990). Multisensory integration requires that stimulus information from multiple modalities converge onto a "supramodal" motor map (Edwards et al. 1979). Such convergence is not trivial, given the underlying sensorimotor transformations for different modalities (Andersen and Buneo 2002; Pouget et al. 2002). Sensory and motor maps are not only lateralized in the mammalian brain, but also represent information in different reference frames in a topographic fashion. Consider for example the transformations for saccades to visual or somatosensory

Address for reprint requests and other correspondence: V. N. Buchholz, Radboud Univ. Nijmegen, Donders Institute for Brain, Cognition, and Behavior, PO Box 9104, NL-6500HE Nijmegen, The Netherlands (e-mail: verena.buchholz@donders.ru.nl). stimuli. The transformation for visually guided saccades is relatively straightforward: from a retinotopic to an oculomotor representation, both coded relative to gaze. The transformation for somatosensory saccades from a somatotopic (coded relative to body surface) to an oculomotor representation is more complicated because it depends on both gaze and body posture (Andersen and Buneo 2002; Azanon et al. 2010a; Groh and Sparks 1996; Heed and Roder 2010; Macaluso et al. 2002; Overvliet et al. 2011). For example, if a sensory stimulus is felt on your left hand while it is positioned to the right of gaze in the right visual field (Fig. $1 A$ ), the sensorimotor transformation requires interhemispheric transfer (Azanon et al. 2010a; Heed and Roder 2010; Overvliet et al. 2011). In this case, the representation of the stimulus in a somatosensory map in the right hemisphere has to be remapped into the oculomotor system in the left hemisphere. Such interhemispheric remapping increases reaction times (RTs) relative to intrahemispheric remapping by approximately $90 \mathrm{~ms}$ (Overvliet et al. 2011). During interhemispheric remapping, subjects occasionally look in the wrong direction (Groh and Sparks 1996; Overvliet et al. 2011), presumably due to activation in the wrong motor map.

Given that some body postures require interhemispheric remapping, whereas others do not, how does this influence the efficiency of multisensory integration of a spatially congruent visuotactile stimulus? To address this question, we had subjects make gaze shifts to visual, tactile, or visuotactile stimuli presented to their fingertips with the arms either in a crossed posture, or in the default, uncrossed posture (Fig. 1A).

Consideration of the principles of multisensory integration (for review, see Stein and Stanford 2008) leads to predictions of behavior when the arms are in the crossed position. From a temporal perspective, because the arrival of the remapped tactile input in the correct motor map is delayed due to interhemispheric remapping, the temporal principle predicts little or no multisensory integration, resulting in visual-like RTs. For example, using different stimulus onset asynchronies, Diederich and Colonius (2007) showed negligible multisensory integration if a tactile stimulus is delayed by $50 \mathrm{~ms}$ relative to a visual stimulus; perhaps different body postures impart a similar influence. From a spatial perspective, if the visual and tactile inputs are initially represented in motor maps in the opposite hemispheres, they might inhibit each other via the spatial principle. Although this scenario would predict fewer direction errors, it also predicts longer RTs compared with the unimodal visual condition.

Our results support neither scenario. Instead, comparison of subject performance across posture revealed substantially 
A

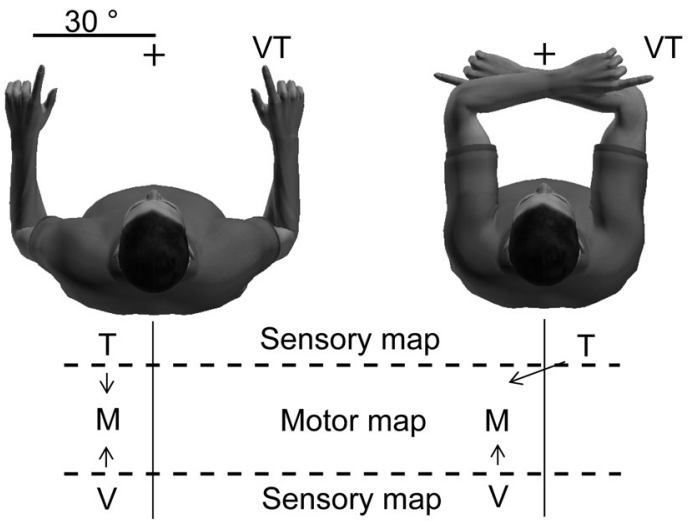

Intra-hemispheric Integration
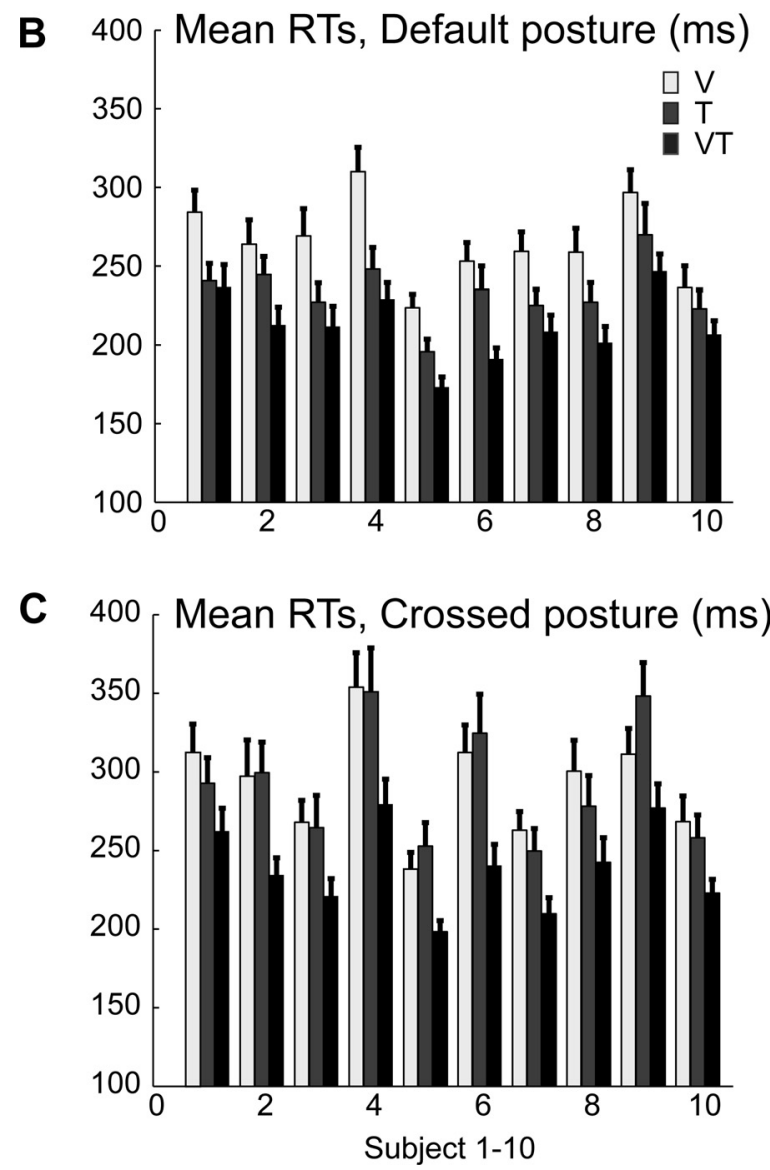

Fig. 1. Experimental design and mean reaction times (RTs) per condition. $A$ : subjects either assumed the default (left) or crossed (right) posture. Depicted below are schematics of two sensory maps (V, visual; $\mathrm{T}$, tactile), feeding into an oculomotor map (M). In the crossed posture, tactile information must be remapped across hemispheres during the sensorimotor transformation. $B$ : mean RTs per participant in the default posture in visual, tactile, and visuotactile trials. The shortest RTs occur in multisensory trials. $C$ : mean RTs per participant in the crossed posture for the three conditions. Again, the shortest RTs occur in multisensory trials.

greater benefits of multisensory integration in the crossed compared with default postures, as measured by RTs. Furthermore, subjects in the crossed posture moved rarely in the wrong direction when presented with visuotactile stimuli, despite the expedited RTs. Our findings reveal an important role for multisensory integration in expediting the accurate completion of complex sensorimotor transformations. These results provide a new perspective on inverse effectiveness observed in multisensory stimuli, which extends beyond external stimulus properties and emphasizes the role of internal spatial representations.

\section{METHODS}

Participants. Ten healthy subjects [ 4 female, mean age 28.1 years (range: 23-38 years), all right-handed] participated in the experiment. None reported any neurological symptoms including sensorimotor dysfunction or neck or back pain. Subjects had either normal or corrected-to-normal vision. All procedures were approved by the Research Ethics Board for Health Science Research at the University of Western Ontario and were in accordance with the Declaration of Helsinki. Subjects gave informed consent and were aware that they could terminate testing at any time.

Setup. Participants sat in a dark room in front of the stimulus device as described elsewhere (Buchholz et al. 2011). Their hands and elbows were placed on a tilted surface of the device, which was located at a distance of $25 \mathrm{~cm}$ in front of the body. The stimulus device was equipped with a set of fiber-optic lights (Omron e3x-na, GB) and piezoelectric Braille stimulators (Metec, Dusseldorf, Germany). Subjects viewed the center of the device with a comfortable, slightly downward gaze direction. The device contained one central optical fiber light, and ten peripheral fiber-optic lights, five on either side, at horizontal eccentricities of $10,16,23,30$, and $36^{\circ}$ (luminance $1.2 \mathrm{~cd} / \mathrm{m}^{2}$; only the $30^{\circ}$ stimuli were used in the present experiment). The hands were placed on the device such that the index fingertips of each hand were aligned with, but just underneath, the peripheral lights at $30^{\circ}$. Each of these fingertips overlaid a piezoelectric Braille stimulation pin. A tactile stimulus was presented to the fingertip by transiently raising this pin with an amplitude of approximately $2 \mathrm{~mm}$, holding it elevated for $50 \mathrm{~ms}$, and then lowering it again. Visual targets were presented for $50 \mathrm{~ms}$. On visuotactile trials, the multisensory stimuli were presented simultaneously. The hands were blocked from vision.

Experimental paradigm. Subjects performed a speeded gaze-orienting task to visual, tactile, or visuotactile stimuli. They were simply instructed to look to the peripheral target as quickly and as accurately as possible, regardless of modality, and were not given any explicit instruction regarding eye-head coordination. The subjects either assumed a crossed-arms posture (requiring interhemispheric remapping of tactile stimuli) or a default arms posture (requiring intrahemispheric remapping; see Fig. 1A). Each trial began with a fixation light presented straight ahead. After a jittered delay (1,000 to $1,350 \mathrm{~ms})$, a stimulus was briefly presented at either the right or the left index finger. The task was performed in complete darkness and bimodal stimuli were always presented spatially aligned. In total 6 blocks ( 3 crossed; 3 default) of 204 trials each were interleaved with the order counterbalanced across subjects, with an equal number of interleaved visual, tactile, and visuotactile trials within each block. In summary, the design consisted of two experimental factors: posture (2 levels) and modality (3 levels). Subjects wore earplugs to prevent hearing any sounds associated with the tactile stimuli.

Data collection and analysis. A detailed description of our methodology is found elsewhere (Goonetilleke et al. 2010). Briefly, horizontal eye movements were measured using bitemporal DC electrooculography, with electrodes placed at the outer canthi of the eyes and six-dimensional head movements were recorded at a rate of $4 \mathrm{kHz}$ using a passive infrared measurement system (MotionMonitor tracker; Innovative Sports Training, Chicago, IL). Furthermore, neck muscle activity was recorded by intramuscular fine-wire needle electrodes in splenius capitis (a neck muscle involved in head turning; see Goonetilleke et al. 2010). Although eye-head coordination does depend on stimulus modality (Populin and Rajala 2011), such patterns 
are not examined in the present study and will be presented elsewhere. Movement onsets and offsets were identified by a computer algorithm that detected velocity crossing thresholds $(50 \%$ for gaze; $10 \%$ for head). These marks were used as guides for interactive marks that could be changed by an analyst within a user interface written in Matlab (The MathWorks, Natick, MA). Trials that were classified offline as anticipatory or too slow due to lack of subject alertness (RTs $<80$ or $>800 \mathrm{~ms}$, respectively) were excluded from further analysis. Trials with secondary saccade amplitudes higher than $50 \%$ of the target eccentricity were left out of further analysis. Fewer than $5 \%$ of trials were excluded using these criteria. Direction errors were categorized into eye-only, head-only, or gaze direction errors if the initial movement direction was incorrect for either the eye, the head, or both, respectively.

\section{RESULTS}

Reaction times. One of the most important benefits of multisensory integration is the shortening of RTs to multimodal stimuli compared with unimodal RTs (for review, see Stein and Stanford 2008). Therefore, we first compared the RTs across unimodal and multimodal conditions for both postures. We pooled across directions after confirming no interactions with our factors of interest with a repeated-measures ANOVA $($ RMA; direction $\times$ modality and direction $\times$ posture and direction $\times$ modality $\times$ posture; all values of $P>$ $0.05)$.

Default posture. Figure $1 B$ shows mean RTs for each modality in the default posture (intrahemispheric remapping). Overall, RTs significantly differed across modality [RMA; $\left.F_{(2,7)}=79.1 ; P<0.05\right]$. RTs to visual stimuli were on average $263 \pm 27 \mathrm{~ms}$ (mean $\pm \mathrm{SD}$ ), which is longer than usually reported, but consistent with presenting a low-intensity visual stimulus at a relatively large eccentricity (Kalesnykas and Hallett 1994). RTs to tactile stimuli averaged $232 \pm 20 \mathrm{~ms}$, which was approximately $30 \mathrm{~ms}$ shorter than RTs to visual stimuli $[t(9)=6.2 ; P<0.05]$. Consistent with the multisensory literature, RTs to visuotactile stimuli were shorter than those to either unimodal stimulus, averaging $209 \pm 21 \mathrm{~ms}$, which was approximately $24 \mathrm{~ms}$ lower than the shortest unimodal RTs $[t(9)=7.7 ; P<0.05$; in the default posture, the tactile stimulus produced the shortest unimodal RTs].

Crossed posture. Figure $1 C$ depicts the mean RTs for each modality in the crossed posture (requiring interhemispheric remapping of tactile stimuli). RTs to visual stimuli were on average $290 \pm 34 \mathrm{~ms}$ and to tactile stimuli $291 \pm 40 \mathrm{~ms}$. Now the crucial question is, what happens if opposite hemispheres receive sensory information from the same stimulus? Despite the crossed posture, RTs to visuotactile stimuli were surprisingly short, averaging $236 \pm 28 \mathrm{~ms}$, which was approximately $55 \mathrm{~ms}$ shorter than the shortest unimodal RTs $[t(9)=11.6 ; P<$ 0.05 ; in the crossed posture, either the visual or the tactile stimulus produced the shortest unimodal RTs].

Comparison of RTs across default vs. crossed postures. To compare the effect of posture more directly, Fig. 2, A-C contrasts visual-only, tactile-only, or visuotactile RTs on a subject-by-subject basis across posture. Somewhat surprisingly, RTs on visual trials increased by $27 \pm 20 \mathrm{~ms}$ in the crossed posture $[t(9)=-3.9 ; P<0.01$; Fig. $2 A]$. This finding suggests an influence of task set and will be investigated in more detail in a later section. RTs to tactile stimuli increased by $59 \pm 26 \mathrm{~ms}$ when subjects adopted a crossed posture
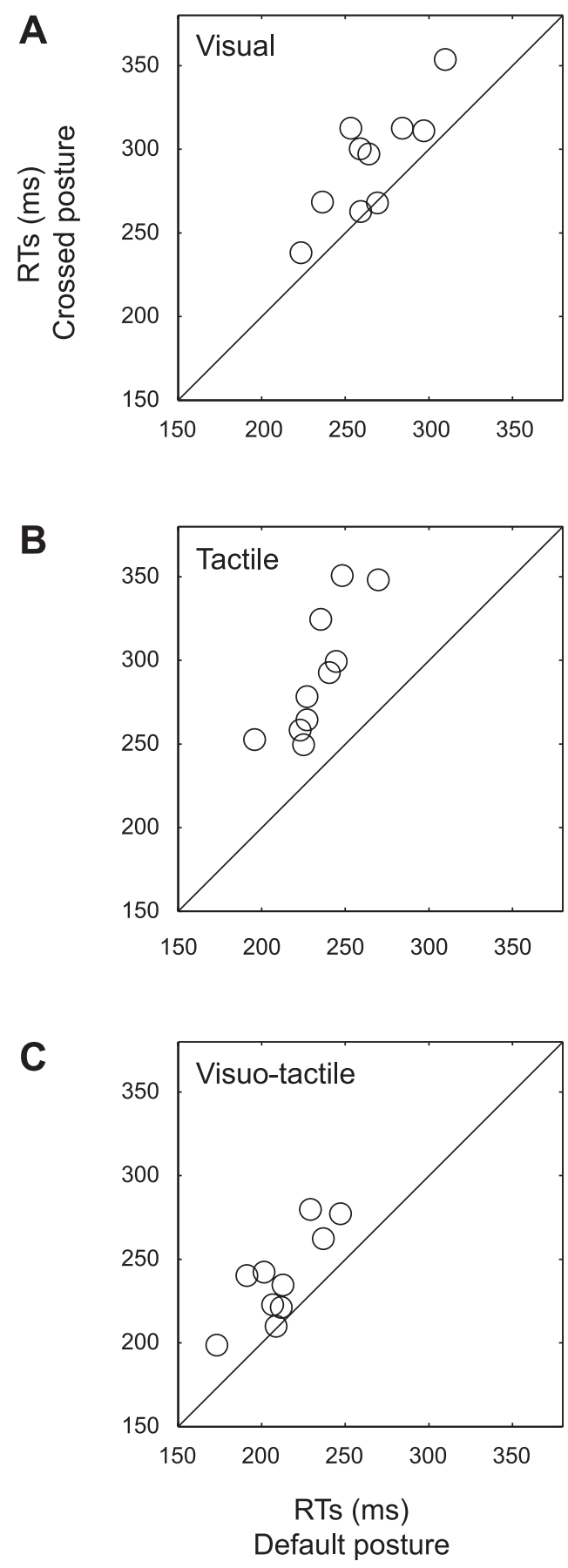

Fig. 2. Direct comparison of RTs across postures. Comparison of participant's mean RTs in the default and crossed posture in visual trials $(A)$, tactile trials $(B)$, or visuotactile trials $(C)$. Each circle shows data from a different subject.

$[t(9)=6.7 ; P<0.05 ;$ Fig. $2 B]$. This finding is consistent with a more complex sensorimotor transformation across hemispheres. Despite this cost associated with interhemispheric transfer, RTs to visuotactile stimuli increased by only $27 \pm 17$ $\operatorname{ms}[t(9)=4.7 ; P<0.05$; Fig. $2 C]$, which is the same as the increase seen for visual-only stimuli.

Greater RT benefits of visuotactile integration in the crossed posture. We conducted two analyses to quantify the benefit on RTs afforded by pairing visual and tactile stimuli in the crossed or default posture. In the first analysis, we calculated the multisensory response enhancement (MRE) index of Rach et 
al. (2011) for each posture. This index quantifies the shortening of the bimodal RTs relative to the shortest unimodal RTs

$$
\mathrm{MRE}=\frac{\min (\mathrm{RTv}, \mathrm{RTt})-\mathrm{RTvt}}{\min (\mathrm{RTv}, \mathrm{RTt})} \times 100 \%
$$

Plotting the MRE calculated on a subject-by-subject for each posture reveals that multisensory integration produced on average a $16 \%$ decrease in RTs in the crossed posture vs. a $9 \%$ decrease in RTs in the default posture $[t(9)=-3.4 ; P=0.05$, Fig. 3A]. Note as well that greater MREs were observed for all 10 subjects in the crossed vs. default posture.

The MRE of Rach et al. (2011) uses the shortest unimodal RT as the benchmark for comparing the benefits of multisensory integration. In a second analysis, we used both unimodal distributions to produce a predicted distribution of multisensory responses, assuming that multisensory responses arise from statistical facilitation of two sensory channels that otherwise remain independent (Colonius and Diederich 2006; Miller 1982; Raab 1962). To construct a multisensory distribution predicted by statistical facilitation, we took the lower of a randomly selected RT drawn from each of the unimodal visual and tactile distributions, and then repeated this step 2,000 times. Using such assumptions, we can quantify across our sample of subjects both the number of observed multisensory RT distributions that lie significantly below those predicted by statistical facilitation, as well as the amount by which the observed distributions fall below the predicted distributions. If the observed multisensory RT distributions are significantly lower than that predicted by statistical facilitation, then this is consistent with the integration of the multisensory stimuli to produce the response; greater deviations from the predicted distributions also suggest a greater degree of integration. The results for this second analysis are shown in Fig. 3, $B$ and $C$. In the default posture, for 7 of 10 subjects visuotactile RTs were significantly shorter than those predicted from statistical facilitation, differing on average by $11 \pm 10 \mathrm{~ms}$ [Fig. $3 B ; t(9)=$ $3.6 ; P<0.01]$. In the crossed posture, visuotactile RTs for all 10 subjects were significantly shorter than those predicted from statistical facilitation, differing on average by $22 \pm 9 \mathrm{~ms}[t(9)=$ 7.3; $P<0.0001]$. Moreover, the difference between the predicted and the observed data was larger for the crossed posture, compared with the default posture $[t(9)=7 ; P<0.0001]$. This difference remains significant if expressed in relative terms, with a mean percentage decrease of $5.2 \%$ vs. $8.3 \%$ for the default and the crossed posture, respectively $[t(9)=4.4 ; P<0.01]$. Thus, even though the crossed posture is associated with the initial activation of sensory channels in different hemispheres and thus requires interhemispheric remapping, RTs to multisensory stimuli in this posture seem to be more facilitated compared with the default posture.

Visuotactile integration in the crossed posture abolishes direction errors. Another important behavioral measure of performance is the reliability of the sensorimotor transformation, in that tactile remapping has been shown to occasionally elicit direction errors (Overvliet et al. 2011). Do the shorter than expected RTs to multisensory stimuli come at the cost of an increased propensity for errors? We accordingly examined the prevalence of errors, looking at both gaze errors, which consist of a coordinated eye-head gaze shift in the incorrect direction (Fig. 4A), and head-only errors, in which the head

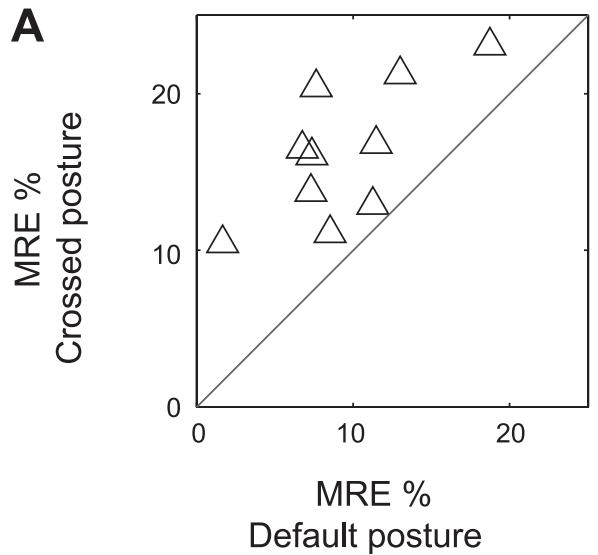

B
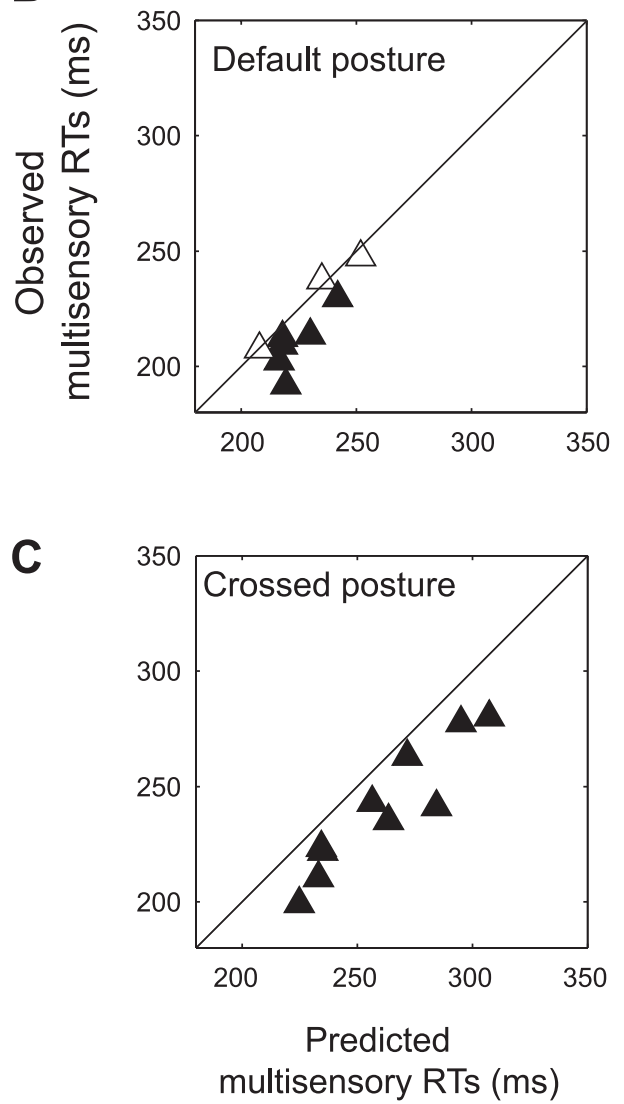

Fig. 3. Measures of the RT benefits from multisensory integration in both postures. A: comparison of multisensory response enhancement values for both postures. $B$ and $C$ : predicted multisensory RTs (under the assumption of statistical facilitation only) vs. observed multisensory RTs in the default $(B)$ or crossed $(C)$ posture. Filled symbols in $B$ and $C$ indicate within-subject significance (two-way $t$-test, $P<0.05$ ).

initially moves in the incorrect direction, but gaze remains stable due to compensatory eye motion prior to a correct eye-head gaze shift (Fig. $4 B$ ). Note that the latter is a more subtle expression of direction errors because it occurs in only one component of the orienting response and gaze behavior is still correct. Both of these error modes have been reported previously in multisensory studies requiring eye-head gaze shifts to misaligned visual-auditory stimuli (Corneil and $\mathrm{Mu}-$ noz 1999b; Corneil et al. 1999a). We rarely observed eye-only 
A

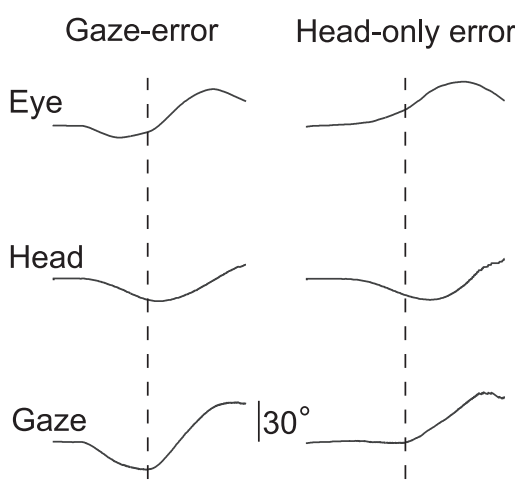

B

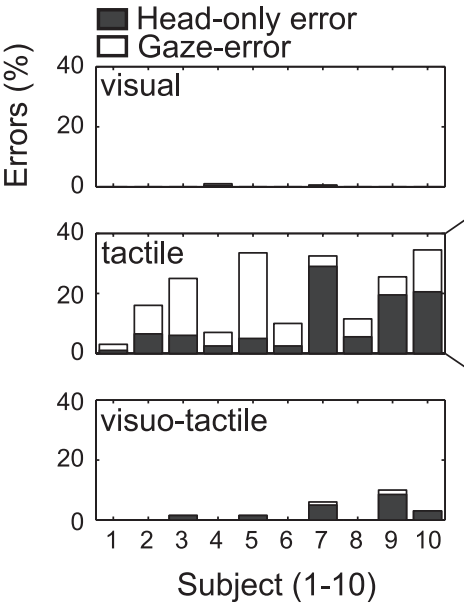

C

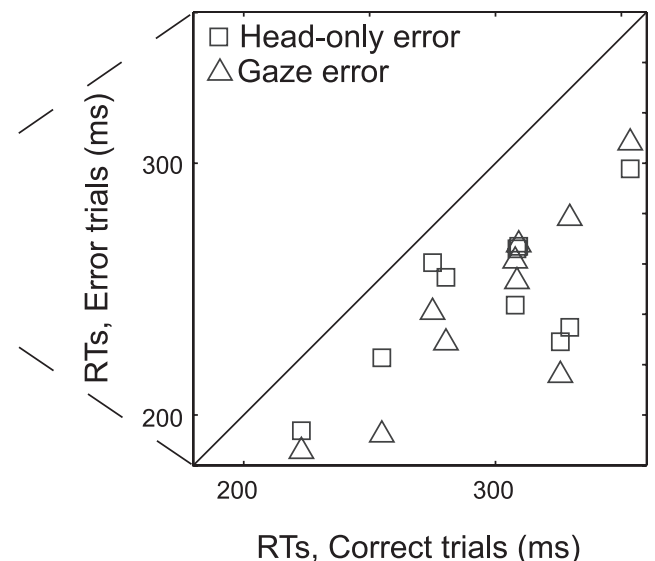

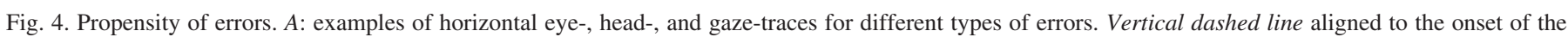

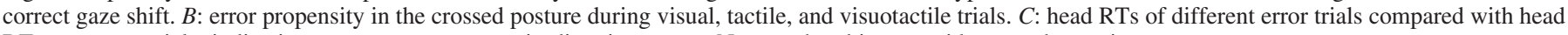
RT on correct trials, indicating premature responses in direction errors. Note each subject provides two data points.

errors ( $<0.1 \%$ of all trials), likely because stimuli were placed beyond the customary eye-only range in humans (Stahl 1999).

Subjects rarely generated either type of error when in the default posture (overall error rate $<1 \%$ ), so we present our analysis of error propensity only in the crossed posture. On visual trials, subjects almost never generated either type of error (Fig. 4B). In agreement with previous results (Overvliet et al. 2011), subjects produced a substantial proportion of errors following the presentation of a tactile-only stimulus with the hands in the crossed posture. Across our sample, either a gaze error or a head-only error was generated on $19.8 \pm 11.7 \%$ of all such trials (range: $3-34.5 \%$ ). As shown in Fig. $4 B$, the type of error produced by a given subject was somewhat idiosyncratic, with the proportion of gaze errors (in white) averaging $10 \pm 8.2 \%$ (range: $2-28.5 \%$ ), and the proportion of head-only errors (in black) averaging $9.8 \pm 9.5 \%$ (range: $1-29 \%$ ). The RTs of the erroneous head movement in both types of errors were significantly shorter $(-52.7 \pm 21.5 \mathrm{~ms} ;-49.7 \pm 28.1$ ms, respectively) than the RT for the head movement accompanying correct eye-head gaze shifts, shown in Fig. $4 C[t(9)=$ 7.7; $P<0.001 ; t(9)=5.6 ; P<0.001]$. This observation is consistent with the notion that errors represent a premature response initiated prior to the completion of the appropriate sensorimotor transformation.

Surprisingly, despite the propensity for errors to tactile-only stimuli in the crossed posture (Fig. $4 B$ ), and the expedited RTs for correct gaze shifts to visuotactile stimuli in the crossed posture (Fig. 1C), subjects generated few direction errors when presented with visuotactile stimuli in the crossed posture (Fig. $4 B)$. Across our sample, the rate of either error type was $2.2 \pm$ $3.3 \%$ (range: $0-10 \%$ ). Most of these errors were head-only movements $(1.9 \pm 2.8 \%$; range: $0-8.5 \%)$, with gaze errors occurring far more rarely than in the tactile-only condition $(0.25 \pm 0.54$; range: $0-1.5 \%)$. Moreover, errors to visuotactile stimuli were generated by those five subjects most prone to errors on tactile-only trials. Interestingly, on average, head RTs of correct visuotactile trials were in the range of the head RTs into the wrong direction in the tactile-only condition $(P>$ 0.10). This analysis emphasizes that a second benefit of pairing visual and tactile stimuli with the arms crossed is an overall reduction in error propensity, and an almost complete abolishment of gaze errors, despite the expedited RTs.

Dynamic response adjustments after tactile-only trials in the crossed posture. Based on the observation of errors, it seems reasonable to assume that an underlying conflict between movement tendencies occurs when tactile input is provided in the crossed posture. Previous research has shown that conflict not only can delay the RT on the current trial, but it can also lead to adjustments in the next trial (Fecteau and Munoz 2003). We therefore wondered if some of the increase in the RT of visual trials in the crossed vs. default posture (Fig. 2A) was due to the previous trial, even if the previous trial was executed correctly. Interestingly, we found a significant increase in the RT of visual trials preceded by correctly performed tactile trials by on average $25 \mathrm{~ms}$ compared with those preceded by visual trials $[t(9)=4.4 ; P<0.01]$. No such increases were found in the default posture (all values of $P>0.10$ ), indicating that this effect is not simply a modality switch cost. Visual trials preceded by visuotactile trials showed a trend toward increased RTs compared with those preceded by visual trials $(\sim 9 \mathrm{~ms})$, although this increase did not reach significance $[t(9)=2.1 ; P=0.06]$. This analysis suggests that at least a portion of the increase in visual RTs in the crossed vs. default posture (Fig. 2A) is due to the effects of the previous trial. Interhemispheric remapping of activity in tactile trials presumably engenders a conflict that has subtle effects on subsequent trials, even if such conflict did not produce an observable error.

\section{DISCUSSION}

In this study, we manipulated body posture to investigate the integration of visual and tactile stimuli for eye-head gaze shifts. Body posture is a fundamental component of any tactilemotor transformation, and our manipulation enabled the direct comparison of multisensory integration during intra- vs. interhemispheric remapping of tactile input. Surprisingly, we found evidence for a greater benefit of multisensory integration in the crossed posture, as indexed by RTs decreases. Moreover, in the crossed posture, such shortening of RTs to multisensory stimuli did not come at the cost of increased error propensity. Overall, these results emphasize a previously unrecognized 
capacity for visuotactile integration to aid the rapid and accurate completion of complex sensorimotor transformations.

Influence of body posture on unimodal responses. Compared with the default posture, gaze shifts in the more complex crossed posture to tactile stimuli had longer RTs and were more prone to errors, consistent with previous reports (Overvliet et al. 2011). Previous studies have suggested that tactile localization consists of an initial somatotopic mapping, which is dynamically integrated some 100-140 ms later with postural information to provide a spatial signal in external or gazecentered coordinates (Azanon and Soto-Faraco 2008; Heed and Roder 2010). Further aspects of our results, specifically the prevalence of gaze and head-only errors in the crossed posture during premature responses, suggest that the early somatotopic mapping of the tactile stimulus in one hemisphere initiates activity on the wrong side of the oculomotor system, prior to the integration of postural information. Such rapid access to oculomotor areas emphasizes a parallel nature to the transformation for gaze shifts to tactile stimuli in the crossed posture.

Our results also show that errors to tactile stimuli presented in complex postures are not simply limited to errors of the visual axis, but can also lead to head-only errors, where gaze is stabilized by compensatory eye movements. We view these subtle head-only errors as complementary to the curved saccades reported by Overvliet and colleagues (2011). Head movements without gaze shifts have also been reported in a visual-auditory distractor paradigm (Corneil and $\mathrm{Munoz}$ $1999 b$ ), during the cancellation of eye-head gaze shifts (Corneil and Elsley 2005) and at low levels of oculomotor activity (Corneil et al. 2002a, 2010; Goonetilleke et al. 2011; Rezvani and Corneil 2008). Together, these studies suggest that headonly errors are initiated at lower levels of oculomotor activity than gaze errors.

Although visually guided gaze shifts should not have been influenced by our postural manipulation, RTs to visual targets increased in the crossed posture. Although a portion of this increase may be due to a different task set associated with the crossed posture, an analysis of after-trial effects demonstrated an increase in visually guided RTs on trials following correctly performed tactile-guided trials. Our interpretation is that this result attests to conflict between competing movement tendencies even on correctly performed tactile trials, complementing the head-only or gaze errors observed on other trials.

To summarize, our postural manipulation influenced both the RTs and error rates of tactile-guided saccades. The nature of such influences provides some insight into the dynamic interhemispheric processing of tactile information in the somatosensory and oculomotor systems. Specifically, we suggest that the presentation of a tactile stimulus while subjects are in a crossed posture initiates activity on the wrong side of the oculomotor system. This initial activation is occasionally strong enough to elicit an overt head-only or gaze error. On other trials, such activity is superseded by activation on the opposite side of the oculomotor system to drive the correct gaze shift, although this transformation takes time. Even on such correctly performed trials, a subtle signature of the conflict inherent to the interhemispheric processing of tactile information is seen in the increased RT on the next trial.

Although we extended previous findings on the dynamics of tactile sensorimotor transformations in a crossed posture, the main focus of this study was on the integration of such information across senses.

Visuotactile integration in the crossed body posture. The results of many studies regarding visuotactile integration have shown that cross-modal interactions take current posture into account, providing evidence for spatially specific cross-modal influences, despite the use of different sensory reference frames (Heed and Roder 2010; Kennett et al. 2001, 2010; Schicke and Roder 2006; Spence et al. 2004; Yamamoto and Kitazawa 2001). This means that the brain has access to postural information, and cortical structures like the posterior parietal cortex (PPC) have been shown to be a key player in integrating postural with sensory information (Azanon et al. 2010b). However, cross-modal effects are usually smaller when the arms are crossed (Kennett et al. 2002; Maravita et al. 2002; Spence and Walton 2005; Wolf et al. 2011) and early mapping mechanisms $(<60-80 \mathrm{~ms})$ do not take posture into account (Azanon and Soto-Faraco 2008; Overvliet et al. 2011). In line with this, and based on the delays inherent to interhemispheric remapping, we had predicted that multisensory integration would be less effective for the crossed posture than that for the default posture, due to the temporal principle. Further, the spatial principle had predicted reduced errors at the cost of increased RTs due to interhemispheric inhibition. Instead, gaze shifts to visuotactile trials were both accurate (i.e., few errors) and rapid (i.e., shorter RTs than expected). In fact, the RTs on correctly performed visuotactile trials were as short as the RTs of errors on tactile-only trials. Thus, subjects in the crossed posture were able to rapidly and reliably integrate multisensory stimuli, suggesting that multisensory integration is more effective under these circumstances than in the default posture.

In what follows, we describe a plausible mechanism to explain these results, which is based on dynamic processing in a multisensory map involved in orienting, the superior colliculus (SC). We acknowledge that the SC is not the only structure where signals from different modalities are integrated, because tactile, proprioceptive, and visual inputs also converge in the frontal eye field, PPC, and premotor cortex (Avillac et al. 2005; Buchholz et al. 2011; Graziano et al.; Thura et al. 2008). Furthermore, the remapping of tactile information into spatial coordinates does not necessarily require saccade planning (Schicke and Roder 2006; Shore et al. 2002; Yamamoto and Kitazawa 2001) and requires input from PPC (Azanon et al. 2010 b). Frontoparietal circuits undoubtedly play a role in resolving the conflict arising from the crossed posture, but the propensity for head-only errors in our task implicates a coordinated interplay with structures close to the motor output. Given this, the SC is an ideal structure with which to illustrate our mechanism (Fig. 5).

Reduction in error propensity. Our speculation that the tactile stimulus in our task initiates activation in the "wrong" side of the oculomotor system is key for the reduction in error propensity in visuotactile vs. tactile trials. Although such activation is weak (after all, errors occur on only approximately $20 \%$ of all tactile trials), its initial oculomotor representation (e.g., within the SC) enables interactions with the oculomotor representation of the visual stimulus. Theoretical and experimental studies have suggested that interactions within the SC are governed by topographically dependent mechanisms enforcing local excitation and distal inhibition 

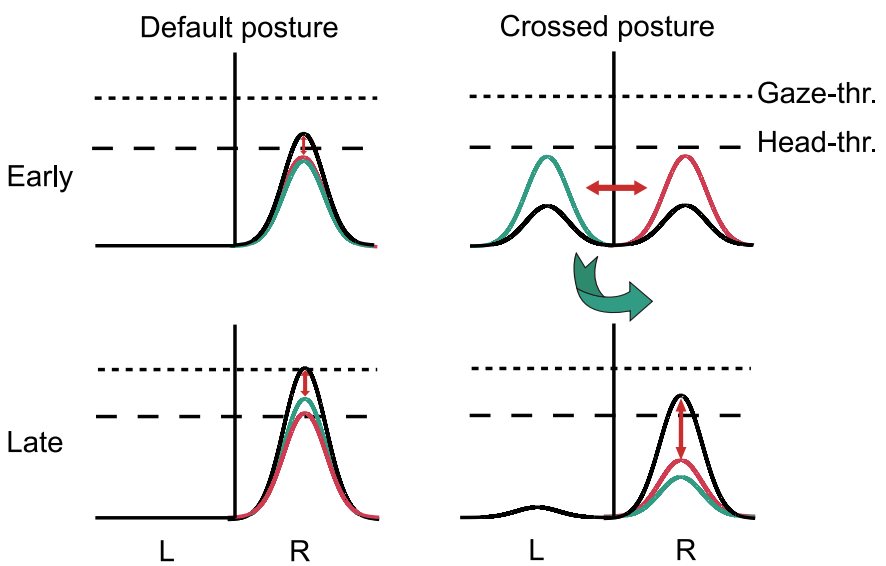

Fig. 5. Conceptual schematic illustrating the interactions between unisensory inputs (visual:red; tactile:green) within the bilateral SC, for both postures These interactions are shown as snapshots either early or late after stimulus presentation. Within each snapshot, the lower horizontal dashed line illustrates the lower threshold for initiating head movements vs. gaze shifts, and the black curve shows the profile of SC activity on visuotactile trials. In the crossed posture, visual and tactile information arrives in opposing SC, leading to mutual inhibition (horizontal red arrow) that produces depressed responses in both SC. The tactile information is subsequently remapped dynamically to the other SC (green arrow), as it is being integrated with postural information via frontoparietal circuits. Although initially weak in the crossed posture, the tactile and visual inputs in the correct SC interact more strongly than they do in the default posture, because of inverse effectiveness (vertical red arrows). Thus, although visuotactile RTs in the default posture are shorter than those in the crossed posture in absolute terms (i.e., SC activity reaches the gaze threshold in the default posture), the relative improvements to behavior are greater in the crossed posture.

(Arai and Keller 2005; Dorris et al. 2007; Grossberg et al. 1997; Marino et al. 2011; McPeek and Keller 2002; Meredith and Ramoa 1998; Munoz and Istvan 1998; Sooksawate et al. 2011; Takahashi et al. 2010), potentially by mechanisms of divisive normalization across the population (Ohshiro et al. 2011). We speculate that weak activation of opposing SC shortly after presentation of visuotactile stimuli in the crossed posture leads to mutual inhibition via the spatial principle (Fig. 5 , top right). Mutual inhibition of these initial sensory-driven responses on visuotactile trials can explain both the reduced propensity for direction errors and the biasing of such direction errors to head-only responses, since head-only errors appear to be initiated at lower levels of oculomotor activity (see above). However, this does not explain the expedited RTs for visuotactile trials in the crossed posture.

Greater RT benefits in the crossed vs. default posture. Recall our speculation that the tactile information in the crossed posture is being dynamically integrated with postural information to enable remapping on the appropriate SC. Such interhemispheric remapping is not required in the uncrossed posture. Because of this, the tactile inputs to the correct SC are likely weaker, at least initially, in the crossed vs. default posture. The principle of inverse effectiveness states that stronger multisensory interactions occur with weaker sensory inputs (Corneil et al. 2002b; Diederich and Colonius 2004; Meredith and Stein 1986); perhaps inverse effectiveness strengthens the integration of visual information with the initially weak tactile representation at the correct SC location (Fig. 5, bottom right). Furthermore, multisensory representations (in this case emerging in the correct SC) are substantially more resistant to competitor-induced depression than are unisensory responses and show stronger noise reduction (Pluta et al. 2011). This multisensory representation would then strengthen over time, as postural information is integrated to resolve the appropriate motor command.

Thus, we propose that a combination of mutual inhibition via the spatial principle and inverse effectiveness early during the tactile remapping process could explain how multisensory integration can shorten RTs and reduce error propensity during complex sensorimotor transformations. Studying multisensory integration during complex sensorimotor transformations at the level of the single neuron clearly requires neurophysiological studies in awake preparations, but it is important to remember that such integration may itself be additionally influenced by the demands on the behavioral task (Bell et al. 2003, 2005; Populin and Yin 2002). Thus, our proposed mechanism might apply only to a speeded response task.

Are alternative scenarios possible? For example, since the visual signal directly leads to veridical activity in the correct location of the oculomotor map, might subjects be completely relying on visual information on such trials? This interpretation cannot explain the far shorter visuotactile vs. visual RTs observed in the crossed posture; clearly tactile information is being integrated somehow. Could subjects, however, be using the spatial information from the visual stimulus and the temporal information from the tactile stimulus (i.e., be using the tactile stimulus as a nonspatial warning cue)? This scenario is inconsistent with a small propensity for direction errors being left in visuotactile trials, and the trend toward after-trial slowing observed following visuotactile trials; apparently the tactile stimulus is still conveying some spatial information.

Inverse effectiveness in resolving complex sensorimotor transformations. A central principle of multisensory integration is inverse effectiveness: the benefits of integration are greater for less effective stimuli. This principle has been observed at a single-cell level (Meredith and Stein 1986) and at a behavioral level (Corneil et al. 2002b; Diederich and Colonius 2004), typically studied by manipulating external stimulus properties. Our results suggest another perspective on how multisensory integration can improve behavior, being more effective for internal spatial representations that are more complex given certain body postures. This perspective has clear relevance beyond visuotactile behavior (Pouget et al. 2002), given the internal coding of sensory stimuli in different reference frames (e.g., eye-centered for vision, head-centered for audition and vestibular sensations, body-centered for somatosensation) and the requirement for convergence into a common motor representation.

\section{ACKNOWLEDGMENTS}

We thank Tobias Heed for helpful comments on the manuscript.

\section{GRANTS}

This work was supported by the Canadian Institutes of Health Research Operating Grant MOP \#93796, the Donders Centre for Cognition, a Discovery grant from the Natural Sciences and Engineering Research Council of Canada, and a travel grant from the Prins Bernhard Cultuurfonds.

\section{DISCLOSURES}

No conflicts of interest, financial or otherwise, are declared by the author(s). 


\section{AUTHOR CONTRIBUTIONS}

V.N.B., W.P.M., and B.D.C. conception and design of research; V.N.B. and S.C.G. performed experiments; V.N.B., W.P.M., and B.D.C. analyzed data; V.N.B., W.P.M., and B.D.C. interpreted results of experiments; V.N.B. prepared figures; V.N.B., W.P.M., and B.D.C. drafted manuscript; V.N.B., W.P.M., and B.D.C. edited and revised manuscript; V.N.B., S.C.G., W.P.M., and B.D.C. approved final version of manuscript.

\section{REFERENCES}

Andersen RA, Buneo CA. Intentional maps in posterior parietal cortex. Аnпи Rev Neurosci 25: 189-220, 2002.

Arai K, Keller EL. A model of the saccade-generating system that accounts for trajectory variations produced by competing visual stimuli. Bio Cybern 92: 21-37, 2005.

Avillac M, Deneve S, Olivier E, Pouget A, Duhamel JR. Reference frames for representing visual and tactile locations in parietal cortex. Nat Neurosci 8: 941-949, 2005.

Azanon E, Camacho K, Soto-Faraco S. Tactile remapping beyond space. Eur J Neurosci 31: 1858-1867, 2010a.

Azanon E, Longo MR, Soto-Faraco S, Haggard P. The posterior parietal cortex remaps touch into external space. Curr Biol 20: 1304-1309, 2010b.

Azanon E, Soto-Faraco S. Changing reference frames during the encoding of tactile events. Curr Biol 18: 1044-1049, 2008.

Bell AH, Corneil BD, Munoz DP, Meredith MA. Engagement of visual fixation suppresses sensory responsiveness and multisensory integration in the primate superior colliculus. Eur J Neurosci 18: 2867-2873, 2003.

Bell AH, Meredith MA, Van Opstal AJ, Munoz DP. Crossmodal integration in the primate superior colliculus underlying the preparation and initiation of saccadic eye movements. J Neurophysiol 93: 3659-3673, 2005.

Buchholz VN, Jensen O, Medendorp WP. Multiple reference frames in cortical oscillatory activity during tactile remapping for saccades. J Neurosci 31: 16864-16871, 2011.

Colonius H, Diederich A. The race model inequality: interpreting a geometric measure of the amount of violation. Psychol Rev 113: 148-154, 2006.

Corneil BD, Elsley JK. Countermanding eye-head gaze shifts in humans: marching orders are delivered to the head first. J Neurophysiol 94: 883-895, 2005.

Corneil BD, Elsley JK, Nagy B, Cushing SL. Motor output evoked by subsaccadic stimulation of primate frontal eye fields. Proc Natl Acad Sci USA 107: 6070-6075, 2010.

Corneil BD, Hing CA, Bautista DV, Munoz DP. Human eye-head gaze shifts in a distractor task. I. Truncated gaze shifts. J Neurophysiol 82: 1390-1405, 1999a.

Corneil BD, Munoz DP. Human eye-head gaze shifts in a distractor task. II. Reduced threshold for initiation of early head movements. J Neurophysiol 82: 1406-1421, 1999b.

Corneil BD, Olivier E, Munoz DP. Neck muscle responses to stimulation of monkey superior colliculus. II. Gaze shift initiation and volitional head movements. J Neurophysiol 88: 2000-2018, 2002a.

Corneil BD, Van Wanrooij M, Munoz DP, Van Opstal AJ. Auditory-visual interactions subserving goal-directed saccades in a complex scene. $\mathrm{J} \mathrm{Neu}$ rophysiol 88: 438-454, 2002b.

Diederich A, Colonius H. Bimodal and trimodal multisensory enhancement: effects of stimulus onset and intensity on reaction time. Percept Psychophys 66: 1388-1404, 2004.

Diederich A, Colonius H. Modeling spatial effects in visual-tactile saccadic reaction time. Percept Psychophys 69: 56-67, 2007.

Dorris MC, Olivier E, Munoz DP. Competitive integration of visual and preparatory signals in the superior colliculus during saccadic programming. J Neurosci 27: 5053-5062, 2007.

Edwards SB, Ginsburgh CL, Henkel CK, Stein BE. Sources of subcortical projections to the superior colliculus in the cat. J Comp Neurol 184: 309-329, 1979.

Fecteau JH, Munoz DP. Exploring the consequences of the previous trial. Nat Rev 4: 435-443, 2003.

Goonetilleke SC, Doherty TJ, Corneil BD. A within-trial measure of the stop signal reaction time in a head-unrestrained oculomotor countermanding task. J Neurophysiol 104: 3677-3690, 2010.

Goonetilleke SC, Gribble PL, Mirsattari SM, Doherty TJ, Corneil BD. Neck muscle responses evoked by transcranial magnetic stimulation of the human frontal eye fields. Eur J Neurosci 33: 2155-2167, 2011.

Graziano MS, Hu XT, Gross CG. Visuospatial properties of ventral premotor cortex. J Neurophysiol 77: 2268-2292, 1997.
Groh JM, Sparks DL. Saccades to somatosensory targets. I. Behavioral characteristics. J Neurophysiol 75: 412-427, 1996.

Grossberg S, Roberts K, Aguilar M, Bullock D. A neural model of multimodal adaptive saccadic eye movement control by superior colliculus. J Neurosci 17: 9706-9725, 1997.

Heed T, Roder B. Common anatomical and external coding for hands and feet in tactile attention: evidence from event-related potentials. J Cogn Neurosci 22: 184-202, 2010.

Kalesnykas RP, Hallett PE. Retinal eccentricity and the latency of eye saccades. Vision Res 34: 517-531, 1994.

Kennett S, Eimer M, Spence C, Driver J. Tactile-visual links in exogenous spatial attention under different postures: convergent evidence from psychophysics and ERPs. J Cogn Neurosci 13: 462-478, 2001.

Kennett S, Rorden C, Husain M, Driver J. Crossmodal visual-tactile extinction: modulation by posture implicates biased competition in proprioceptively reconstructed space. J Neuropsychol 4: 15-32, 2010.

Kennett S, Spence C, Driver J. Visuotactile links in covert exogenous spatial attention remap across changes in unseen hand posture. Percept Psychophys 64: 1083-1094, 2002.

Macaluso E, Frith CD, Driver J. Crossmodal spatial influences of touch on extrastriate visual areas take current gaze direction into account. Neuron 34: 647-658, 2002.

Maravita A, Spence C, Kennett S, Driver J. Tool-use changes multimodal spatial interactions between vision and touch in normal humans. Cognition 83: B25-B34, 2002.

Marino RA, Trappenberg TP, Dorris M, Munoz DP. Spatial interactions in the superior colliculus predict saccade behavior in a neural field model. $J$ Cogn Neurosci 24: 315-336, 2012.

McPeek RM, Keller EL. Superior colliculus activity related to concurrent processing of saccade goals in a visual search task. J Neurophysiol 87: 1805-1815, 2002.

Meredith MA, Ramoa AS. Intrinsic circuitry of the superior colliculus: pharmacophysiological identification of horizontally oriented inhibitory interneurons. J Neurophysiol 79: 1597-1602, 1998.

Meredith MA, Stein BE. Spatial factors determine the activity of multisensory neurons in cat superior colliculus. Brain Res 365: 350-354, 1986.

Miller J. Divided attention: evidence for coactivation with redundant signals. Cogn Psychol 14: 247-279, 1982.

Munoz DP, Istvan PJ. Lateral inhibitory interactions in the intermediate layers of the monkey superior colliculus. J Neurophysiol 79: 1193-1209, 1998.

Ohshiro T, Angelaki DE, DeAngelis GC. A normalization model of multisensory integration. Nat Neurosci 14: 775-782, 2011.

Overvliet KE, Azanon E, Soto-Faraco S. Somatosensory saccades reveal the timing of tactile spatial remapping. Neuropsychology 49: 3046-3052, 2011.

Pluta SR, Rowland BA, Stanford TR, Stein BE. Alterations to multisensory and unisensory integration by stimulus competition. J Neurophysiol 106: 3091-3101, 2011.

Populin LC, Rajala AZ. Target modality determines eye-head coordination in nonhuman primates: implications for gaze control. J Neurophysiol 106: 2000-2011, 2011.

Populin LC, Yin TC. Bimodal interactions in the superior colliculus of the behaving cat. J Neurosci 22: 2826-2834, 2002.

Pouget A, Deneve S, Duhamel JR. A computational perspective on the neural basis of multisensory spatial representations. Nat Rev 3: 741-747, 2002.

Raab DH. Statistical facilitation of simple reaction times. Trans NY Acad Sci 24: 574-590, 1962.

Rach S, Diederich A, Colonius H. On quantifying multisensory interaction effects in reaction time and detection rate. Psychol Res 75: 77-94, 2011.

Rezvani S, Corneil BD. Recruitment of a head-turning synergy by lowfrequency activity in the primate superior colliculus. J Neurophysiol 100: 397-411, 2008.

Schicke T, Roder B. Spatial remapping of touch: confusion of perceived stimulus order across hand and foot. Proc Natl Acad Sci USA 103: 1180811813, 2006.

Shore DI, Spry E, Spence C. Confusing the mind by crossing the hands. Brain Res 14: 153-163, 2002.

Sooksawate T, Isa K, Behan M, Yanagawa Y, Isa T. Organization of GABAergic inhibition in the motor output layer of the superior colliculus. Eur J Neurosci 33: 421-432, 2011.

Spence C, Pavani F, Driver J. Spatial constraints on visual-tactile crossmodal distractor congruency effects. Cogn Affect Behav Neurosci 4: 148169, 2004. 
Spence C, Walton M. On the inability to ignore touch when responding to vision in the crossmodal congruency task. Acta Psychol 118: 47-70, 2005.

Stahl JS. Amplitude of human head movements associated with horizontal saccades. Exp Brain Res 126: 41-54, 1999.

Stein BE, Meredith MA. Multisensory integration: neural and behavioral solutions for dealing with stimuli from different sensory modalities. Ann NY Acad Sci 608: 51-70, 1990.

Stein BE, Stanford TR. Multisensory integration: current issues from the perspective of the single neuron. Nat Rev 9: 255-266, 2008.

Takahashi M, Sugiuchi Y, Shinoda Y. Topographic organization of excitatory and inhibitory commissural connections in the superior colliculi and their functional roles in saccade generation. J Neurophysiol 104: 31463167, 2010.

Thura D, Hadj-Bouziane F, Meunier M, Boussaoud D. Hand position modulates saccadic activity in the frontal eye field. Behav Brain Res 186: 148-153, 2008.

Wolf CC, Ball A, Ocklenburg S, Otto T, Heed T, Roder B, Gunturkun $\mathbf{O}$. Visuotactile interactions in the congenitally acallosal brain: evidence for early cerebral plasticity. Neuropsychology 49: 3908-3916, 2011.

Yamamoto S, Kitazawa S. Reversal of subjective temporal order due to arm crossing. Nat Neurosci 4: 759-765, 2001.

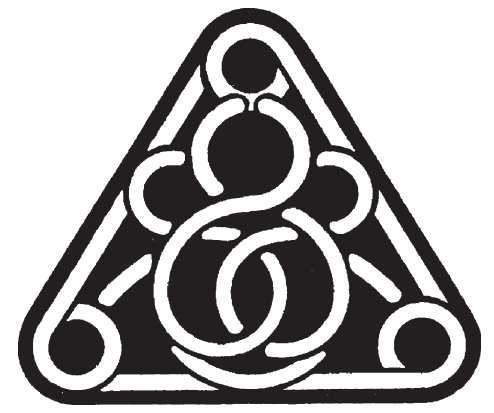

